\title{
Control Strategy and Algorithm Study on Light Vehicle Electronic
}

\section{Mechanical Braking System}

\author{
Wu Xinyu \\ Automotive Engineering institute, Jiangxi University of Technology, Nanchang 330098, China
}

\section{Keywords: Control Strategy; Light Vehicle; Electronic Mechanical Braking System}

Abstract. When the vehicle is braking, its gravity center will move forward, and the rear wheel traction is reduced, so is the braking force, resulting in the braking distance longer. If it's more serious, it will also result in tail wagging phenomenon. If the rear wheel braking force can be increased at this time, it will not only shorten the braking distance, but also ensure the stability of automobile braking. EMB is the inevitable trend of automotive brake systems; therefore, how to apply the existing vehicle control algorithms, such as ABS, TCS, ESP and others for EMB becomes one issue that the automotive engineers shall solve. This paper describes the basic principles, hardware components and design methods of EMB, and introduces ABS + EBD braking control algorithm based on EMB, to verify its feasibility.

\section{Introduction}

With the continuous development and improvement of automotive technology, automotive design introduces a number of electronic control systems such as ABS, TCS, ESP, etc. while improving performance, making the traditional structure and piping arrangements of hydraulic or air brakes more complex, assembly and the difficulty of maintenance increasing, hydraulic (air) circuit leakage risks becoming higher. More importantly, the conventional hydraulic brake system cannot meet the modern car requirements of simple precise control and rapid response. In the past 10 years, the western countries rose the development of the study on vehicle-wire system (x-by-wire), and the wire control brake system (brake-by-wire) came into being, which launched on electro-mechanical brake (Electromechanical Brake) study. Simply speaking, electro-mechanical brake is to change the original hydraulic or pneumatically driver parts into electric motor to drive, in order to improve response time and increase braking efficiency and so on, but also greatly simplifies the structure, reducing the assembly and maintenance difficulty. It replaces traditional braking system with electronic brake pedal and electric drive actuator, and adjusts braking force according to the driver's intentions, the road and vehicle state comprehensively to achieve optimum braking performance and improve vehicle active safety.

As a new generation braking system, EMB has unparalleled advantages compared with conventional hydraulic systems. First, EMB cancels the brake master cylinder, hydraulic lines, etc., which greatly simplifies the braking system structure, facilitating the arrangement, equipping and maintenance; assembly and test use modular structures, and the electrical interface are more robust and reliable without mechanical lever through the division plate, and so it improves driving safety. And because it does not use brake fluid, environmental pollution was reduced. Secondly, replacing hydraulic components with mechanical motor makes EMB control simple and accurate with faster 
response. Once receiving the order, the brakes on each wheel can produce large enough force in a very short period, shortening the braking time so that the braking distance will be greatly reduced.

At the same time, highly integrated electronic control components make EMB system software upgrades and update easy. If you want to integrate other control system function, just simply add appropriate sensors and control algorithms, such as EBD, ABS, TCS, ESP, EPB (electronic parking braking), ACC (adaptive cruise control). After adopting electronic brake pedal, the EMB pedal characteristics can be adjusted accordingly depending on different models and the driver habit. Also under ABS mode, the pedal cannot perform rebound and vibration, almost no residual brake torque and noise brake comfort will be significantly improved.

However, EMB system that in the experimental stage still faces many problems to be solved. A large number of electronic components that used in EMB inevitably have certain failure or power failure. It is the primary problem to be solved about how to address these problems timely to ensure the driving safety. Therefore, the system needs to adopt more redundancy and fault-tolerant technology. Also from the point of vehicle driving safety, the braking system will produce a lot of heat under high speed braking, which will make the working environment very bad and test the performance and stability under high temperature conditions of EMB drive motor and other components. The anti-electromagnetic interference ability of large number of electronic circuits in handling complicated driving conditions has become one of the key technologies for EMB system design. Considering from the production cost, due to the use of a large number of sensors, control chip and meet the requirements of new generation of car communication system, EMB will need to install a compatible and more advanced communication protocol (such as Flexray or TTCAN) communication elements, which makes the current EMB cost higher than the conventional hydraulic brake system, thus reducing the system cost is also a problem to be solved currently. From the view of vehicle design, EMB wheel braking force actuator increases the unsprung masses, which affect vehicle performance and increase the arrangement difficult.

\section{ABS and EBD control principle and their advantages}

EBD begins to control the braking force when the car is braking, while ABS begins to work when the wheel appears locking tendency, which is the essential difference of ABS control and EBD control. Both ABS and EBD make control on the wheel torque, to prevent the wheels from sliding relative to the road surface, in order to take full advantage of road adhesion coefficient and prevent the vehicle out of control caused by different road adhesion coefficient of both sides causing additional steering torque. Although ABS can guarantee the stability of the rear wheels, but the comfort for ABS working is bad. The EBD only uses slip rate without using the wheel deceleration to detect wheel locking tendency. Comparing with ABS, the threshold of EBD slip ratio is lower, and the increasing or decreasing gradient of brake pressure adjustment is significantly lower, with priority of considering holding pressure. It has good braking comfort, so it's called "highly comfortable rear axle ABS". EBD still has advantages of achieving best braking effect on different roads, to shorten braking distances and improve braking sensitivity and coordination.

Comparing with ABS, EBD has no additional hardware, but only control procedures and functional optimization and enhancement, or even we can say that EBD is a derived auxiliary function from ABS. By improving and enhancing software control logic within the ABS computer, operation function is more complicated, which can be identified in some automotive products manual with $\mathrm{ABS}+\mathrm{EBD}$. In addition to adding certain control program in preparing the required computing 
program, automotive engineers don't have too much hardware investment. EBD can allocate braking force intelligently according to motion state of each wheel to maintain vehicle balance, smooth and direction under braking state. Moreover, even if ABS failed, EBD can also ensure the vehicle will not be overturned due to spin. Currently, the China-made cars AudiA6, Odyssey, Picasso, Palio, Mazda Familia, Elantra have adopted EBD as standard security configuration to improve product performance. So EBD does not need too much investment installed, and now most of the domestic low-mid-range cars are also equipped with this function.

Another feature of EBD is its mobility. When the load or the number of occupants in the vehicle change, EBD can still perform "intelligent" distribution actively, timely and reasonably of braking force based on each wheel speed sensor signal, so as to ensure straight running state of the vehicle and the stability of vehicle body, to kill the risks in the bud.

\section{Integrated Control Algorithm}

ABS and EBD control is not very significant since the car speed is lower when the car can be controlled by the driver, so the setting in the program is when the vehicle speed is less than $2 \mathrm{~m} / \mathrm{s}$, braking force output shall be achieved according to the displacement output applied by the driver on the brake pedal.

For initial braking, we take front wheel maximum slip ratio $\mathrm{Sf}$ as the threshold to determine entering the braking control, and the maximum slip ratio Smax of four wheels as judging condition to enter ABS control. From the initial braking, the vehicle system module begins to calculate the wheel slip rate, and the slip rate starts at zero, with EBD module automatically running. When the maximum slip ratio of four wheels is greater than 0.2, change the ABS flag bit ABS_Flag as 1 and enter into the ABS control as well. At the same time, EBD and ABS module has only one function, and the target control amount of these two modules are the target clamping force of EMB actuator, inputting the current wheel slip ratio.

According to the existing literature, there are two control algorithms based on EBD of EMB here:

1)The rear wheel slip rate shall be controlled in the vicinity of a predetermined value of the front wheel slip ratio. When the rear wheel slip ratio is greater than a predetermined value of the front wheel slip ratio, the rear wheels should be remained pressure. At the same time, the front wheel braking pressure is proportional to the output of pedal perception, the diagram of EBD control subroutine is prepared based. Wherein sr is rear wheel slip ratio, sf is front-wheel slip ratio, Flag_s is rear wheel control threshold. When the current front wheel slip rate is greater than Flag_sf, increase the output of rear wheel brake pressure rapidly. When the current slip rate is less than Flag_sf, increase the rear wheel braking pressure output slowly. The aim is to enable rapid growth of the rear wheel slip rate curve in the beginning, and then relatively steady growth, remaining the vicinity of certain percentage below the front wheel slip curve in order to take full advantage of the adhesion coefficient of the vehicle. Because in actual simulation we find that the single pressure adjustment speed gives up the adhesion coefficient of the rear wheel when ensuring the curve stability, so the braking performance is not ideal.

2)When the difference between the rear wheel speed and the front wheel speed is greater than or equal to a predetermined threshold (eg, 0.01), remain pressure of the rear wheel, while pressurizing the front wheel; on the contrary, pressurize the rear wheel while holding pressure for the front wheels. The aim is to increase the reaction speed of system. The foresaid program is discussed with threshold control method, which may also adopt PID, fuzzy control and other intelligent control methods to 
control. Through the demonstration, the second method is better than the first in the control speed and accuracy, but the algorithm correctness is based on the correctness of front and rear wheel speed calculation, which is unattainable in practice. So the EBD subroutine prepared by the first strategy is used in this paper,.

\section{Vehicle running dynamics model}

Multivariant vehicle system model should be established in order to make evaluations for the handling stability and braking performance of automotive brake system under specific conditions. To reduce the complexity of the model, we need to simplify the actual vehicle model:

1. Coordinate origin of the vehicle coordinate system coincides with the vehicle center of gravity;

2. The vehicle pitch angle around the $y$-axis and roll angle around the $x$-axis are zero;

3. Ignore the role of suspension system, and the vehicle has no vertical movement;

4. The same mechanical properties of the tires;

5. The steering wheel angle is proportional to the front wheel angle and two front wheel angles are the same;

6. Ignore wind drag resistance, wheel rolling resistance and driveline friction torque during braking.

In this paper, seven degrees of freedom vehicle models are adopted, including the vehicle longitudinal displacement, lateral displacement, yaw angular velocity and rotation of four wheels. The input includes longitudinal force, lateral force, wheel steering angle and brake braking torque of each wheel, and the output is the state of vehicle motion. Simplify vehicle dynamics model as shown in Fig. .1. The geodetic coordinate system and the vehicle coordinate system are represented by XOY, xoy respectively.

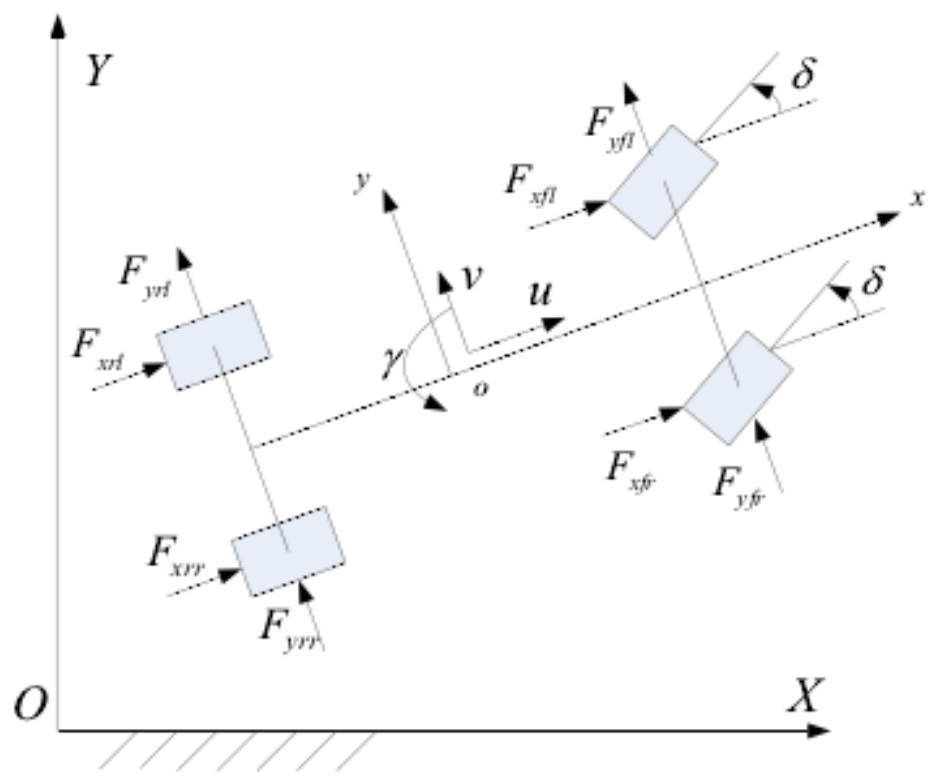

Figure 1. Dynamic model

\section{Results}

From the simulation results, we can see that when the ABS or ABS + EBD braking control take effect, braking distance or braking time is less than the controlled, which is mainly because when 
there is no control, the front wheels are locked and dragged to slip that rolling wheel resistance becomes sliding resistance, affecting the braking performance. More importantly, when the rear wheels are locked, the front wheels are not locked, the vehicle is easy to slip, which is dangerous working conditions and should be avoided.

By comparing the case 2, case 3 simulation parameters, we find that $\mathrm{ABS}+\mathrm{EBD}$ integrated brake control time is 6.6 percent more than ABS control braking time, braking distance increasing 6.3 percent, but $\mathrm{ABS}+\mathrm{EBD}$ control can strictly limit wheel slip ratio within a stable region to ensure the safety. The control strategy only with ABS control can control wheel slip rate around $20 \%$ in order to obtain the shortest possible braking distance and braking time.

EMB is the inevitable trend of automotive brake system development, therefore, how apply the existing vehicle control algorithms, such as ABS, TCS, ESP and others for EMB has become one issue of the automotive engineers' to solve. This paper describes the basic principles, hardware components and design methods of EMB, and provides braking control algorithm based on EMB $\mathrm{ABS}+\mathrm{EBD}$, verifying its feasibility.

\section{Acknowledgements}

This work was financially supported by the key subject building project (vehicle engineering) of Jiangxi University of Technology.

\section{References}

[1] Hannan M A, Azidin F A, Mohamed A. Hybrid electric vehicles and their challenges: A review[J]. Renewable and Sustainable Energy Reviews, 2014, 29: 135-150.

[2] Torres J L, Gonzalez R, Gimenez A, et al. Energy management strategy for plug-in hybrid electric vehicles. A comparative study[J]. Applied Energy, 2014, 113: 816-824.

[3] Bovee K, Rizzoni G, Midlam-Mohler S, et al. Well-to-wheel analysis and measurement of energy use and greenhouse gas and criteria emissions in a Plug-in Hybrid Vehicle: the EcoCAR 2 case study[C]//Electric Vehicle Conference (IEVC), 2014 IEEE International. IEEE, 2014: 1-8.

[4] Lv C, Zhang J, Li Y, et al. Hardware-in-the-loop simulation of pressure-difference-limiting modulation of the hydraulic brake for regenerative braking control of electric vehicles[J]. Proceedings of the Institution of Mechanical Engineers, Part D: Journal of Automobile Engineering, 2014: 0954407013516942.

[5] Chiang W P, Yin D, Omae M, et al. Integrated Slip-Based Torque Control of Antilock Braking System for In-Wheel Motor Electric Vehicle[J]. IEEJ Journal of Industry Applications, 2014, 3(4): 318-327.

[6] Xu L, Li J, Ouyang M, et al. Multi-mode control strategy for fuel cell electric vehicles regarding fuel economy and durability[J]. International Journal of Hydrogen Energy, 2014, 39(5): 2374-2389.

[7] Wang B, Xu M, Yang L. Study on the economic and environmental benefits of different EV powertrain topologies[J]. Energy Conversion and Management, 2014, 86: 916-926.

[8] Slough S, Goossens P, Schwarz C, et al. Virtual Prototyping of Electric Drive Systems for System-Level Parameter Studies and Optimization[R]. SAE Technical Paper, 2014. 
[9] Kong C L, Wan M S, Chen N, et al. Modeling and Simulation of Electro-Hydraulic Compound Regenerative Braking Control Strategy for Electric Vehicle[C]//Applied Mechanics and Materials. 2015, 701: 733-738.

[10]Chen C. The development of hybrid electric vehicle control strategy based on GT-SUITE and Simulink[C]//2015 International Conference on Intelligent Systems Research and Mechatronics Engineering. Atlantis Press, 2015. 